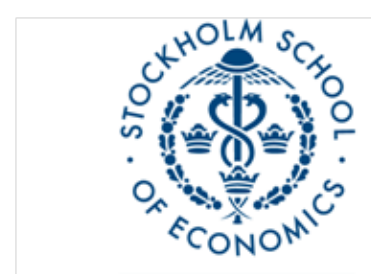

SITE STOCKHOLM INSTITUTE OF

TRANSITION ECONOMICS

SITE Working Paper No. 07, year 2010

\title{
Paying Attention to Payoffs in Analogy-based Learning
}

\section{Topi Miettinen}

Stockholm Institute of Transition Economics

Stockholm School of Economics Sveavägen 65, P.O. Box 6501 SE - 11383 Stockholm 


\title{
Paying Attention to Payoffs in Analogy-based Learning*
}

\author{
Topi Miettinen ${ }^{\dagger}$ \\ SITE, Stockholm School of Economics
}

February 2, 2010

\begin{abstract}
This paper introduces the payoff-confirming analogy-based expectation equilibrium (PCABEE) as a way to refine the set of analogy-based equilibria and the associated admissible analogy partitions. In addition to the actions of others, own payoff history provides information about others' strategies but, yet, non-Bayesian Nash equilibria may exist both with an incorrect and a correct prior. We provide general conditions when this happens. Two stylized employer-employee interactions, one with a correct and one with an incorrect prior, are provided illustrating how PCABEE can be used to analyze robust stereotypes and how incorrect such stereotypes may lead to discrimination.
\end{abstract}

KEYWORDS: analogy expectations, bounded rationality, curse, learning, discrimination, stereotypes

JEL: C72, D82

\section{Introduction}

Analogy-based expectations equilibrium (Jehiel, 2005; Jehiel and Koessler, 2008) provides a powerful tool to understand how steady-state behavior may differ from Nash equilibrium behavior when players use stereotypical classifications, analogy classes, when learning about others. Stereotypes must be confirmed by

\footnotetext{
*Thanks to Philippe Jehiel for comments and encouragement. Thanks also to Drew Fudenberg for comments on an earlier draft. All errors are mine. Financial support of the Yrjö Jahnsson Foundation gratefully acknowledged.

${ }^{\dagger}$ Sveavägen 65, PO Box 6501, SE-11383 Stockholm, Sweden; topi.miettinen@hhs.se
} 
experience. Not only the observed behavior of others, but also own successes and failures when interacting with them, provide data to test one's stereotypical beliefs. Along these lines, this paper introduces the Payoff-confirming analogybased expectations equilibrium to refine the set of analogy-based expectations equilibria and the associated admissible analogy partitions.

The analogy-based expectation equilibrium is related to and can be considered as a self-confirming equilibrium (Fudenberg and Levine, 1993; Fudenberg and Levine, 1995; Dekel et al. 2004) and conjectural equilibrium (Battigalli, 1987). Thus, it is based on the implicit assumption that over time each player gains experience about the characteristics and behavior in the opponent population(s). Each player records which actions are chosen and which characteristics prevail in that population(s). When observing the opponents, each player classifies opponents according to some characteristics, whether strategically and informationally relevant or not. She then organizes samples of observed actions into these classes (called analogy classes), one sample for each class of opponent characteristics, and expects that the opponents with the characteristics of the class play the sample average strategy of the class.

The opponents' characteristics used for sample classification may differ from others' strategically relevant characteristics, their types. If the sample classification partitions opponents more coarsely than the opponents' types, this may naturally lead to an underestimation of the correlation between opponents' types and actions.

Stereotyping on gender, clothing or ethnicity provide examples of analogy classifications which typically miss the strategically relevant aspects of the characteristics although they may capture some coarse correlations of characteristics and strategies.

One may argue, as Fryer and Jackson (2008) do, that memory limitations may necessitate categorical or stereotypical classifications. ${ }^{1}$ Their concern is more on illustrating how such coarse stereotyping and discrimination of minorities may come about as one tries to minimize prediction errors. The point of this paper is to take categorical simplifications on opponent types as given and study when they can survive in a equilibrium when cateogory marginals on action choices, on the one hand, and marginals on own payoffs, on the other hand, are kept track of and used to test one's stereotypical beliefs in a strategic interaction setting.

\footnotetext{
${ }^{1}$ See also Mohlin (2009).
} 
Our two examples in Section 2 illustrate how stereotyping on gender may lead to gender discrimination in equilibrium although gender is entirely payoffirrelevant and although own success experiences are used to test one's stereotypes in equilibrium. ${ }^{2}$

Arguably, people often observe and remember how successful they have been over time when learning about interacting with others. When modeling learning with stereotypes, it is thus often plausible to assume that players have at least a coarse track-record of their own payoffs. The successes and the failures are what players care for - if they do not, why should they best-reply in the first place. Thus, the failures and successes tend to be remembered. If the sampled payoffs differ from the distribution that the player expects, given her beliefs about opponent types and actions, she should eventually realize the inconsistency and abandon her incorrect conjectures. The payoff samples thus provide a natural way to study the robustness of the analogy classification and the corresponding equilibrium.

Two considerations should be kept in mind here. First, a player needs a large amount of data to test his conjectures, i.e. whatever data is used when testing, it must be restored in memory over time. Thus data on payoffs, as relevant success experiences, may be used when testing conjectures although own types are not used because the latter are not rememebered over time. ${ }^{3}$ Second, although success experiences are used to test conjectures, they may be only coarsely recalled (as are opponent types). Payoff realizations in broader intervals may be coded into a single payoff class when testing conjectures. Both these considerations suggest that recalling pwn payoffs does not necessarily imply a very demanding test on one's stereotypical conjectures.

A weak robustness check requires that the conjectured marginal payoff distribution must be consistent with the marginal sample payoff distribution. Assuming that players carry out a more sophisticated consistency check would require careful consideration: when one is interested in implications of underappreciation of correlation between types and actions, one would beg the question by requiring beliefs to be consistent with the sample joint marginals of own

\footnotetext{
${ }^{2}$ Our explanation of gender discrimination differs markedly from previous explanations (Arrow, 1998; Loury, 1998; Mailath et al., 2000). In previous models, statistical discrimination is justified through correct conjectures whereas here discrimination is due to incorrect stereotypes or misperception of strategically relevant variables.

${ }^{3}$ This may happen when own type varies from interaction to another and thus is not part of inherent personality characteristics and does not remain the same over the entire learning process.
} 
payoffs and opponent actions, for instance ${ }^{4}$.

This paper studies such a weak refinement, the payoff-confirming ABEE (PCABEE) with one primary question in mind: when is an ABEE payoffconfirming also? Clearly, if an ABEE coincides with a Bayesian-Nash equilibrium, it must be payoff-confirming also since conjectures in a Nash equilibrium are correct. The challenge is then to identify and study cases where a PCABEE can differ from Bayesian-Nash equilibrium.

We first show by means of an example that there are PCABEE which differ from Bayesian-Nash equilibria even when the prior is correct. We then provide general sufficient conditions for an ABEE with a correct prior to be payoffconfirming. These conditions are also necessary if one requires robustness to perturbations of the prior or if one confines attention to two-player games with two actions and two states. Since a Bayesian-Nash equilibrium is a PCABEE, the latter always exists when the former does. In particular, a PCABEE always exists in finite environments.

Analogy-based expectation equilibrium has been shown to provide a lacking learning justification ${ }^{5}$ for the cursed equilibrium (Eyster and Rabin, 2005; Jehiel and Koessler, 2008; Miettinen, 2009). Esponda (2008) also studies the learning foundations of the cursed equilibrium assuming that players learn others' actions and their own payoffs and that they occasionally learn something about the opponents' characteristics. He illustrates that in the steady states of such learning (i) there is even less trade than in the Nash equilibrium in bilateral common value trade, (ii) players bid less aggressively in common value auctions and (iii) less effort is provided in team work. This is to be contrasted with Eyster and Rabin's (2005) result that there is more trade in the cursed equilibrium than in a Nash equilibrium of the bilateral trade, for instance.

We show that Esponda's conclusions hold also in the present context by pointing out that his equilibria correspond to particular PCABEE. As Esponda informally conjectured, the fact that observing payoffs leads to stronger selection problems hinges upon an incorrect prior assumption. We show that when players have correct conjectures about the others' types, then learning own payoffs in addition to opponents' actions and types implies that the set of Payoff-confirming analogy-based equilibria collapses to the set of Bayesian-Nash

\footnotetext{
${ }^{4}$ Dekel, Fudenberg and Levine (2004) consider such refinements in contexts with more sophisticated players who keep track of their own types and actions in addition to other signals and perfectly understand correlations.

${ }^{5}$ See Fudenberg (2006).
} 
equilibria even when players fail to keep track of the correlations per se. This is rather intuitive: in the setups with monotone payoffs studied by Esponda, players can make sharp inferences based on payoffs.

In non-monotone setups, however, even payoff-confirming ABEE with a correct prior may have incorrect strategy conjectures. This is illustrated in section 2 by a stylized workplace relationship example. For this to hold, it is crucial either that in each analogy-class, given opponent characteristics, every positive probability equilibrium action of others gives the same payoff, or that, given each positive probability action of others, the payoff is the same for each positive probability characteristics of the other. Such conditions, of course, are hardly satisfied in strict monotone setups, where higher types of others are associated with higher own types and thus higher own payoffs given an action profile of the opponents.

The paper is organized as follows. In Section 2, two examples on gender stereotyping are provided which both induce a reason for discrimination in equilibrium. In Section 3 the model is presented. In Section 4, it is assumed that the prior is known and a quite general class of games is analyzed. It is shown that generally there are PCABEE that differ from BNE and conditions are provided when this is the case. In Section 5 the games with monotone selection studied by Esponda are considered. The results are discussed in Section 6 .

\section{Motivating examples}

\subsection{Workplace relationship}

Let us consider the following simple two-player game (see Figure 1). The row player is an employer and the column player can be either a male or a female employee, $g \in M, W$ respectively. Most of the time due to well-planned incentive schemes perhaps, the interests of the employer and the employee are aligned. In the state of aligned interests, $\theta_{g}^{A}$ occurring with probability $p_{g}^{A}=p^{A}$ for $g=M, W$, the parties thus play a game with a unique (Nash) equilibrium, $(T, R)$, where the equilibrium outcome is the preferred one for both. Sometimes however, i.e. in state $\theta_{g}^{C}$ with $p_{g}^{C}=p^{C}$ for $g=M, F$, the interests of the employer and the employee are entirely conflicting and they play a constant sum game with the only (Nash) equilibrium in mixed strategies,

$\sigma_{1}(T)=1 / 2, \sigma_{2}(L)=3 / 4$. It is known to both whether interests are aligned or not and thus the above state-conditioning strategies constitute the unique Nash- 
equilibrium of the game. Moreover, the probability that interests are aligned is the same for men and women.

\begin{tabular}{|l|l|l|}
\hline$\theta_{g}^{A}$ & $L$ & $R$ \\
\hline$T$ & 2,2 & 4,4 \\
\hline$B$ & 1,1 & 3,3 \\
\hline
\end{tabular}

\begin{tabular}{|l|l|l|}
\hline$\theta_{g}^{C}$ & $L$ & $R$ \\
\hline$T$ & 3,2 & 1,4 \\
\hline$B$ & 2,3 & 4,1 \\
\hline
\end{tabular}

Figure 1: Stereotyping at workplace relationship, $g=1,2$

The two payoff matrices with aligned and conflicting interests are given in Figure 1. Notice that the matrices do not depend on gender. Yet, let the employer bundle together states of aligned and conflicting interests when gathering experiences of playing with women whereas he finely observes and classifies the behavior of men. Formally, the employer has a unique analogy class for women, $\left\{\theta_{W}^{A}, \theta_{W}^{C}\right\}$, whereas he has two separate analogy classes to organize his observations of the choices of men, $\left\{\left\{\theta_{M}^{A}\right\},\left\{\theta_{M}^{C}\right\}\right\} .{ }^{6}$ This may be due to diverted attentiveness when playing with women or due to a belief that female employees are unable to detect the strategic aspects of the situation. Since the employer adopts the simplest belief that all types in a given class behave indentically, the employer ends up conjecturing that females always behave similarly in both states whereas males may play a separation strategy.

Can such stereotypes survive in equilibrium? Can they be confirmed by experiences? This test is central in the analogy-based expectations equilibrium where the analogy classes express the stereotypes, and where players are assumed to learn each others actions over time and adopt only stereotypes consistent with this experience.

The following ABEE illustrates that gender stereotypes, for instance, can strongly influence equilibrium play despite the payoff irrelevance. Male employees play $\mathrm{R}$ in $\theta_{M}^{A}$ and the Nash-equilibrium mixed strategy in $\theta_{M}^{C}, \sigma_{2}\left(L \mid \theta_{M}^{C}\right)=$ $3 / 4$. Female employees play $\mathrm{R}$ in $\theta_{W}^{A}$ and $\mathrm{L}$ in $\theta_{W}^{C}$. Thus, the employer believes that male employees play the Nash-equilibrium strategy whereas female employees, in each state, play $\mathrm{R}$ with probability $p^{A}$ and $\mathrm{L}$ with probability $p^{C}$. Best-responding to the believed strategy of a female employee amounts to

\footnotetext{
${ }^{6}$ For simplicity, let each employee finely bundle the employer's choices into two singleton analogy classes, one for $\theta_{g}^{A}$ and the other for $\theta_{g}^{2}$. This implies that the employee always has correct conjectures about the strategy of the employer.
} 
playing $\mathrm{T}$ in $\theta_{W}^{A}$ and $\mathrm{B}$ in $\theta_{W}^{C}$ for any prior probability of state $A$ greater than $1 / 4$. It is easy to see that playing $\mathrm{L}$ is indeed a best-response strategy of a female employee in state $\theta_{W}^{C}$. The remaining best-responses are easily verified as they are Nash-equilibrium strategies. Thus, we have an equilibrium.

Notice that the expected payoff of the employer is lower in $\theta_{W}^{C}$ when facing a female employee than when facing a male, $\theta_{M}^{C}$. In $\theta^{C}$, the expected payoff in the mixed strategy equilibrium played against a male is

$$
\begin{aligned}
4 p^{A}+ & p^{C}\left[3 \sigma_{1}\left(T \mid \theta_{M}^{C}\right) \sigma_{2}\left(L \mid \theta_{M}^{C}\right)+\sigma_{1}\left(T \mid \theta_{M}^{C}\right) \sigma_{2}\left(R \mid \theta_{M}^{C}\right)\right. \\
& \left.+2 \sigma_{1}\left(B \mid \theta_{M}^{C}\right) \sigma_{2}\left(L \mid \theta_{M}^{C}\right)+4 \sigma_{1}\left(B \mid \theta_{M}^{C}\right) \sigma_{2}\left(R \mid \theta_{M}^{C}\right)\right] \\
= & 4 p^{A}+5 / 2 p^{C}
\end{aligned}
$$

whereas the payoff when playing a female gives only $4 p^{A}+2 p^{C}$, giving a motive for discrimination. Thus, even the payoffs themselves confirm the employer's gender stereotypes. The actual marginal payoff distribution (aggregating over states) coincides with what the employer expects to receive. In particular when facing a female, the employer's payoff is 2 with probability $p^{A}$ and his payoff is 4 with probability $p^{C}$.

Stereotypes that are in such a manner robust to payoff information are probably particularly deeply grounded. This example illustrates that a boundedly rational player who expects types and strategies in each analogy-class to be non-correlated, may not Bayesian-Nash best-respond even if she observes the payoffs and even if she knows the prior distribution of types. The purpose of the current paper is to study which stereotypes, and under which circumstances, are robust to using payoff information to challenge one's stereotyped beliefs.

Crucial in our example is that, for female employees, once a choice of the employee is fixed, the payoff is the same in both states given the employer's own equilibrium actions. It will be shown in section 4 , that this type of condition guarantees that a non-Nash analogy-based expectation equilibrium is payoff confirming. Alternatively, in each state of the analogy class, the payoff must be the same whatever positive probability actions in the class the opponents choose. 


\subsection{Bilateral labor market}

Consider a bilateral labor market with an employer, B, and an employee, S. The employer, the row player, wishes to hire the employee which can be a male or a female, $g \in M, W$, and of a low, $q^{L}$, or of a high quality, $q^{H}$, each with probability $1 / 2$ independent of gender. The quality is known to the employee only.

\begin{tabular}{|l|l|l|}
\hline$q_{g}^{H}$ & $H$ & $L$ \\
\hline$H$ & 2,1 & 2,1 \\
\hline$L$ & 0,0 & $3,-1$ \\
\hline
\end{tabular}

\begin{tabular}{|l|l|l|}
\hline$q_{g}^{L}$ & $H$ & $L$ \\
\hline$H$ & $-1,2$ & $-1,2$ \\
\hline$L$ & 0,0 & 1,1 \\
\hline
\end{tabular}

Figure 2: Stereotyping in bilateral labor market

Each party chooses between a high wage and a low wage, $w_{i} \in\{L, H\}$, $i=1,2$. The payoff matrices, independent of gender are given in Figure 2. If the wages are compatible, that is, if $w_{B} \geq w_{S}$, the employee is hired with the wage offered by the employer, $w_{B}$, otherwise not. If the employee is not hired, the employer's payoff is 0 . Hiring a low quality employee with a low wage gives payoff 1 , hiring a high quality employee with a high wage gives 2 and hiring a high quality employee with a low wage gives payoff 3 . On the other hand, hiring a low quality employee with a high wage generates a loss.

Notice that the employer's marginal return of increasing her wage offer is increasing in the quality of the employee. The employee weakly prefers asking a wage that coincides with her quality - that is a low quality employee asks for a low wage and a high quality one asks for a high wage. Assume for simplicity that the employee adopts this weakly dominant strategy. Given this employee strategy, a higher employer offer selects a higher distribution of quality. Thus, the game is one of monotone selection studied by Esponda (2008).

If the employer knows this strategy (and the exogenous quality distribution), it is optimal for the employer to offer a high wage. This is her Bayesian-Nash best-response strategy. ${ }^{7}$

Yet, it may be reasonable to assume that the employer knows neither the strategy nor the quality of the employee but rather has to infer the quality and

${ }^{7}$ There is also another Bayesian-Nash equilibrium: $(H, H H)$. 
the average strategy of the employee population from her observations when repeatedly hiring various employees. Then, if the employer always offers a low wage for female employees, she gets only low quality female employees. She observes that half of the female employees ask for a high wage. It is then consistent to believe that there are only low quality female employees in the market and that they demand a high and low wage with equal probability. Offering a low wage is optimal given these beliefs. Moreover, the employer cannot infer from her stream of payoffs that there would be higher quality employees in the market. Thus, bounded perception of the interaction generates a selection problem, less trade and more inefficiencies than in the Bayesian-Nash equilibrium.

Offering a high wage, to male employees, allows the employer to hire both high and low quality male employees leading to correct (Nash-equilibrium) beliefs. The payoffs confirm the belief that high quality males ask for a high wage and low quality males ask for a low one.

This in another example of learning by analogies. The employer expects that the quality that she sees is the true sample of qualities. For female employees, she does not pay attention to the fact that the variation in the employees' actions might be an indication of variation in quality. Rather the employer takes for granted that the distribution of actions represents the actual variation of the behavior of the female employees of the same quality. The payoffs only confirm this belief.

This example is an instance of a more general phenomenon in learning environments with complementarites. The phenomenon was identified by Esponda (2008). We illustrate it in section 5 and illustrate that it holds also in analogybased learning.

\section{Model}

\subsection{The underlying game, strategies and expectations}

The game is a static game of incomplete information, $\left(A_{i} ; u_{i} ; \Theta ; p(\theta) ; i=\right.$ $1, \ldots, N)$. There are $N$ players indexed by $i=1, \ldots, N$. An action of player $i$ is $a_{i}$ and the finite set of actions ${ }^{8}$ available to her is $A_{i}$. The actions of players other than $i$ are denoted by $a_{-i} \in \times_{j \neq i} A_{j}$. An action profile is $a \in A=\times_{i=1}^{N} A_{i}$.

\footnotetext{
${ }^{8}$ The set of actions is the same at every type profile.
} 
Exogenous uncertainty in the stage game is modeled by letting nature draw a type profile, $\theta$, with probability $p(\theta)$ prior to the play of the stage game. The type profile is a vector of types, one for nature and one for each player, $\theta \in$ $\Theta=\times_{i=0}^{N} \Theta_{i}$ where $\theta_{i} \in \Theta_{i}$ is the type of player $i$. Nature may have its own type, $\theta_{0} \in \Theta_{0}$, to allow for cases where the player types alone do not determine payoffs. For simplicity, we suppose that the set of type profiles is finite. The vector of types of players other than $i$ is $\theta_{-i} \in \Theta_{-i}=\times_{j \neq i} \Theta_{j}$. The outcomes are type and action profile combinations, $(a, \theta)$. The payoff depends on the actions and on the type profile: $u_{i}: A \times \Theta \rightarrow R$ for $i=1, \ldots, N$.

A strategy of player $i$ is a function of her type, $\sigma_{i}: \Theta_{i} \rightarrow \Delta\left(A_{i}\right)$ and the probability that type $\theta_{i}$ chooses action $a_{i} \in A_{i}$ is denoted by $\sigma_{i}\left(a_{i} \mid \theta_{i}\right)$. The strategies of players other than $i$ are denoted by $\sigma_{-i}: \Theta_{-i} \rightarrow \times_{j \neq i} \Delta\left(A_{j}\right)$ and a strategy profile is $\sigma: \Theta \rightarrow \times_{j=1}^{N} \Delta\left(A_{j}\right)$. The conjecture of $i$ about the state and about the strategy of the opponents ${ }^{9}$ is denoted by $\widehat{\mu}^{i}: \Theta \rightarrow \Delta(\Theta)$ and $\widehat{\sigma}_{-i}^{i}$ : $\Theta \rightarrow \Delta(A)$, respectively.

Implicit in the model, there is a learning process where, at each round, each player plays against randomly chosen opponents, one player drawn from each opponent population and all randomly matched players then receive a random draw of types, their stage game specific private information. The equilibria in our context are understood as steady states of this learning process. The equilibrium concept of main interest is the payoff-confirming analogy-based expectation equilibrium which assumes that players observe opponent actions, characteristics and own payoffs during the learning process while failing to understand correlations in their samples due to simplified observation or organization of these. This concept is defined in the next subsection.

\subsubsection{Payoff-confirming analogy-based expectation equilibrium}

In the analogy-based expectation equilibrium each player $i$ partitions the support of type profiles, $\Theta$, into analogy classes. Player $i$ 's partition, $\mathcal{A}_{i}$, is called the analogy partition of player $i$. An element of $\mathcal{A}_{i}$ is a set of type profiles denoted by $\alpha_{i}$, the element of $\mathcal{A}_{i}$ containing $\theta$ is $\alpha_{i}(\theta)$. An analogy system $\left(\mathcal{A}_{1}, \ldots, \mathcal{A}_{N}\right)$ describes the partitions of each player $i=1, \ldots, N$. Whereas a player's information partition describes how precisely the player observes the type profile at the interim stage, the analogy partition describes the player's

\footnotetext{
${ }^{9}$ The player may be unaware of the opponent's private information partition. Therefore, the conjecture is conditioned on the whole type profile rather than on the profile of opponents' types.
} 
stereotypes. This translated into how precisely he keeps track of the type profile realizations ex-post when the game is played.

The analogy-based expectation equilibrium is specific about how the conjectures are formed. Each player conjectures that, at a given type profile, each opponent plays her average strategy of the analogy class where that type profile belongs to. This is the simplest theory consistent with observing only the analogy class rather than the precise opponent type. Moreover, this is the only consistent theory where each opponent plays a pooling strategy in each analogy class. To formalize this idea, we define the opponents' average strategy in a set of type profiles $B \subset \Theta$ as follows:

$$
\bar{\sigma}_{-i}(B)=\frac{\sum_{\theta \in B} p(\theta) \sigma_{-i}\left(\theta_{-i}\right)}{\sum_{\theta \in B} p(\theta)} .
$$

We are now ready to define the Analogy-based expectation equilibrium:

Definition 1 The triple $\left(\sigma_{i}, \widehat{\sigma}_{-i}, \mathcal{A}_{i}\right)_{i=1}^{N}$ is an Analogy-based expectation equilibrium with a correct prior if, first, for all $\theta \in \Theta$, for all $i$ and $a_{i}^{*} \in \operatorname{supp}\left[\sigma_{i}\left(\theta_{i}\right)\right]$

$$
a^{*} \in \arg \max \sum_{\theta_{-i} \in \Theta_{-i}} p\left(\theta_{-i} \mid \theta_{i}\right) \sum_{a_{-i} \in A_{-i}} \widehat{\sigma}_{-i}^{i}(\theta) u_{i}(a ; \theta)
$$

and, second, for all $\theta \in \Theta$, for all $i \quad \widehat{\sigma}_{-i}^{i}(\theta)=\bar{\sigma}_{-i}\left(\alpha_{i}(\theta)\right)$.

Moreover, let us denote by $\operatorname{ABEE}_{\mathcal{A}_{i}=\Theta_{i}}\left(\operatorname{ABEE}_{\mathcal{A}_{i} \leq \Theta_{i}}\right)$ an analogy-based expectation equilibrium where each player's analogy partition coincides with (is finer than) the player's information partition. The coarseness of the partitions is part of the equilibrium description rather than exogenous. This is where our concept differs from the definition in Jehiel and Koessler (2008) who assume that the analogy partitions are exogenous.

As an example, consider the workplace relationship of section 2. The employer builds analogies around gender and the nature of the interaction and aggregates data on female employee choices without paying attention to the nature of interaction where each choice was made.

Notice that the above definition implicitly subsumes that the type distribution is known: in the maximization problem, the player expects each type with the correct probability $p$. There are situations where it is not natural to assume that either players know the prior or that they always observe (at least coarsely) the characteristics of the other. It may be then reasonable to assume that the 
expected distribution of characteristics is endogenous. Thus, an ABEE with an incorrect prior is needed.

Definition 2 The triple $\left(\sigma_{i}, \widehat{\sigma}_{-i}, \mathcal{A}_{i}\right)_{i=1}^{N}$ is an Analogy-based expectation equilibrium with an incorrect prior if, first, for all $\theta \in \Theta$, for all $i$ and $a_{i}^{*} \in \operatorname{supp}\left[\sigma_{i}\left(\theta_{i}\right)\right]$

$$
a^{*} \in \arg \max \sum_{\theta_{-i} \in \Theta_{-i}} \widehat{\mu}^{i}\left(\theta_{-i} \mid \theta_{i}\right) \sum_{a_{-i} \in A_{-i}} \widehat{\sigma}_{-i}^{i}(\theta) u_{i}(a ; \theta),
$$

second, for all $\theta \in \Theta$, for all $i, \widehat{\sigma}_{-i}^{i}(\theta)=\bar{\sigma}_{-i}\left(\alpha_{i}(\theta)\right)$ and, third,

for every $\alpha_{i} \in \mathcal{A}_{i}, \sum_{\theta \in \alpha_{i}} p(\theta)=\sum_{\theta \in \alpha_{i}} \widehat{\mu}^{i}(\theta)$.

With an incorrect prior, the conjecture about the distribution of types may be incorrect. Thus, the analogy partition does not necessarily partition the actual set of type profiles. However, the analogy classes are distinct and, for every analogy class in the analogy partition, the sample probability of that class must coincide with the expected probability of that class.

If we model a learning process where the same player plays repeatedly against varying opponents, arguably, each player should observe and remember at least her own payoffs. The successes and the failures are what players care for if they do not, why should they best-reply in the first place. This is what motivates a refinement of the ABEE, the payoff-confirming ABEE (PCABEE). In this section we formally define the concept. The PCABEE studies a mild robustness check of the ABEE, where the player fails to keep track how own payoffs are correlated with other signals that she learns from each previous round of play. Thus, the marginal expected and sample payoff distribution must coincide. It would be unnatural to assume that players perceive correlations between own payoffs and opponents' actions or types although they do not perceive correlations between actions and types in each analogy class.

Let us now define the Payoff-confirming ABEE:

Definition 3 Payoff-confirming analogy-based expectation equilibrium is an ABEE where for all $i$ and $\bar{u}_{i}$

$$
\begin{aligned}
& \sum_{\left\{a \in A, \theta \in \Theta \mid u_{i}\left(a_{i}, a_{-i}, \theta_{i}, \theta_{-i}\right)=\bar{u}_{i}\right\}} \widehat{\mu}^{i}(\theta) \sigma_{i}\left(a_{i} \mid \theta_{i}\right) \widehat{\sigma}_{-i}^{i}\left(a_{-i} \mid \theta\right) \\
& =\sum_{\left\{a \in A, \theta \in \Theta \mid u_{i}\left(a_{i}, a_{-i}, \theta_{i}, \theta_{-i}\right)=\bar{u}_{i}\right\}} p(\theta) \sigma_{i}\left(a_{i} \mid \theta_{i}\right) \sigma_{-i}\left(a_{-i} \mid \theta_{-i}\right) .
\end{aligned}
$$

If the prior is correct, then $\widehat{\mu}^{i}(\theta)=p(\theta)$. 
In a PCABEE, the conjectured probability of each payoff in an analogy class must coincide with the sample probability of that payoff in that class.

\section{Correct prior}

In this section, we study the payoff-confirming equilibria with a correct prior and thus, $\widehat{\mu}^{i}(\theta)=p(\theta)$. Notice that in the workplace relationship example in section 2 , the employer has correct expectations about the nature of situation. The employer bundles together both states when learning about the female employer choices. This leads her to believe that the employee plays a mixed strategy in each state. This in turn, justifies non-best-responding to the actual choice of a female employee when interests are conflicting. The example illustrates that there can be PCABEE (with a correct prior) that do not correspond to Bayesian-Nash equilibria.

Proposition 1 There can be PCABEE with correct prior which are not BayesianNash equilibria.

The main purpose of this section is to identify cases, when this can happen under a correct prior. Clearly, when an ABEE strategy profile is a Bayesian Nash equilibrium, then conjectures about others' strategies must be correct by the definition of the Nash equilibrium. Then surely, if conjectures are correct, payoff-information cannot reveal anything which was not known already. Thus, an ABEE which is Bayesian-Nash must be payoff-confirming also. There are two simple cases when this happens (see also Jehiel and Koessler, 2008): first, the ex-post observation of types more precise than the interim information when strategy is chosen; second, pooling strategies.

Whenever the analogy-expectations are correct, we have a BNE. This may hold even if a player's analogy partition is coarser than some opponent's information partition if the opponent types in such a coarser analogy class play a pooling strategy as expected by the analogy expectations. This property characterizes the Bayesian-Nash ABEE.

Proposition 2 ABEE is BNE iff, for each player, her opponents' types play a pooling strategy in each of the player's analogy classes.

Formally, ABEE is BNE iff $\theta^{\prime} \in \alpha_{i}(\theta)$ implies that $\sigma_{-i}(\theta)=\sigma_{-i}\left(\theta^{\prime}\right)$.

The proposition is stated without a proof but the idea is simple. In at least one analogy partition, two types mus play differently for ABEE to differ from 
BNE. When there are such two types then necessarily the average strategy differs from the actual strategies of these two types and thus the ABEE differs from BNE. Thus only if the analogy partition is coarser than some opponents' information partition and the opponent plays a separation strategy, a PCABEE might differ from a Bayesian-Nash equilibrium. In this case, some non-generic payoffs ${ }^{10}$ are needed in order for a PCABEE to differ from a Bayesian-Nash equilibrium. Otherwise, the payoff information would reveal any mistaken conjectures. In two-player two-action two-state games of incomplete information, it is fairly easy to characterize the set of pure strategy ABEE that are PCABEE.

Proposition 3 Let $N=2, \Theta=\left\{\theta^{1}, \theta^{2}\right\}$ and $A_{i}=\left\{a_{i}^{1}, a_{i}^{2}\right\}$. Suppose that $s$ is a pure strategy ABEE.

The ABEE is payoff-confirming if and only if

- $s$ is a Bayesian-Nash equilibrium

or

- for each $i$ such that $s_{i}\left(\theta^{m}\right) \neq s_{i}\left(\theta^{n}\right)$ and $\mathcal{A}_{j}=\left\{\left\{\theta^{1}, \theta^{2}\right\}\right\}$

$$
\text { for all } m, u_{j}\left(s_{j}\left(\theta^{m}\right), r_{i}\left(\theta^{m}\right) ; \theta^{m}\right)=u_{j}\left(s_{j}\left(\theta^{m}\right), s_{i}\left(\theta^{m}\right) ; \theta^{m}\right)
$$

or

$$
\text { for all } \left.m, u_{j}\left(s_{j}\left(\theta^{m}\right), r_{i}\left(\theta^{m}\right) ; \theta^{m}\right)=u_{j}\left(s_{j}\left(\theta^{n}\right), s_{i}\left(\theta^{n}\right) ; \theta^{n}\right)\right)
$$

where $r_{i}\left(\theta^{m}\right)$ is the action not chosen by $i$ at $\theta^{m}$.

Proof. In the appendix.

To gain some intuition to this result, notice that each player is trying to detect a correlation between opponents' actions and the opponents' type profile using payoff realizations as evidence. If there is evidence for correlation between actions and types, then clearly, the presumption that the opponents play the average strategy in each state of the analogy class must be incorrect. The fact that either condition (5) or condition (6) holds prevents inferring anything about the joint distribution: If condition (5) holds, player's payoff is the same given opponent type whatever the opponent chooses. In other words, there is no strategic uncertainty about own payoffs in each state. Alternatively, if (6) holds,

\footnotetext{
${ }^{10}$ Notice that this may be due to coarse partioning of payoffs. Since this point is straightforward, I have decided to not write it down formally to avoid unnecessary complications of the model.
} 
the payoff is the same whatever the state given the action of the opponent. That is, there is no exogenous uncertainty about own payoffs given the action of the opponent. In either case, the payoffs do not provide any additional information about the joint distribution of actions and types of others.

In games with more states, more players and more actions, conditions parallel to (5) or (6) are sufficient but not necessary for an ABEE to be PCABEE.

Proposition 4 Let in an ABEE $\sigma$ differ from a Bayesian-Nash equilibrium. If for each $j$ and $\alpha_{j}$ such that there are $\theta^{m}, \theta^{n} \in \alpha_{j}$ with $\sigma_{-j}\left(\theta^{m}\right) \neq \sigma_{-j}\left(\theta^{n}\right)$,

- either for all $\theta \in \alpha_{j}$ and for all action profiles of players other than $j$, $a_{-j}^{*}=\left(a_{1}^{*}, \ldots, a_{j-1}^{*}, a_{j+1}^{*}, \ldots, a_{N}^{*}\right)$ such that for each $i \neq j$ there is $\theta^{\prime} \in$

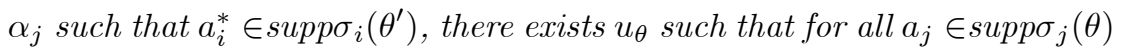

$$
u_{j}\left(a_{j}, a_{-j}^{*} ; \theta\right)=u_{\theta}
$$

- or for all action profiles of players other than $j$, $a_{-j}^{*}=\left(a_{1}^{*}, \ldots, a_{j-1}^{*}, a_{j+1}^{*}, \ldots, a_{N}^{*}\right)$ such that for each $i \neq j$ there is $\theta^{\prime} \in$ $\alpha_{j}$ such that $a_{i}^{*} \in \operatorname{supp} \sigma_{i}\left(\theta^{\prime}\right)$, there exists $u_{a_{-j}^{*}}$ such that for all $\theta \in \alpha_{j}$ and $a_{j} \in \operatorname{suppo}_{j}(\theta)$

$$
u_{j}\left(a_{j}, a_{-j}^{*} ; \theta\right)=u_{a_{-j}^{*}},
$$

then the $A B E E$ is payoff-confirming.

Proof. In the appendix.

Now for any separating strategy and a coarse analogy partition of the opponent such that an opponent plays different strategies in two nodes of an analogy class, either (7) or (8) holds. Again, the former condition aggregates over strategic uncertainty and the latter condition aggregates over exogenous uncertainty. Thus, a player cannot infer anything about the joint distribution of type profiles and action profiles of other players in each analogy class. Notice moreover that proposition 4 does not impose restrictions on the distribution of types. Therefore, a PCABEE satisfying the conditions of the proposition is robust to changes in the distribution of exogenous payoff uncertainty.

Nevertheless, the next example shows that a PCABEE which is not BNE may fail (7) and (8). 
Example 1 There are two players and three states of nature, $\left\{\theta^{1}, \theta^{2}, \theta^{3}\right\}$, each drawn with probability $1 / 3$. This prior is known to both players and the realization of the state is revealed to both. Each state of nature is associated with a simultaneous move two-player game. In each of the games, each player has three actions, $A_{i}=\left\{a^{1}, a^{2}, a^{3}\right\}$. Player two gets payoff +1 if he matches the state, $u_{2}=1$ if $a_{2}=a^{k}$ and $\theta^{k}$ is drawn by nature, and -1 if his action does not match the state. Payoffs of player one are indicated in matrices below.

\begin{tabular}{|c|l|l|l|}
\hline$\theta^{1}$ & $a^{1}$ & $a^{2}$ & $a^{3}$ \\
\hline$a^{1}$ & 1 & 0 & -1 \\
\hline$a^{2}$ & 2 & -2 & -2 \\
\hline$a^{3}$ & 2 & -2 & -2 \\
\hline
\end{tabular}

\begin{tabular}{|l|l|l|l|}
\hline$\theta^{2}$ & $a^{1}$ & $a^{2}$ & $a^{3}$ \\
\hline$a^{1}$ & -1 & 0 & -1 \\
\hline$a^{2}$ & 0 & -1 & 1 \\
\hline$a^{3}$ & -1 & 0 & -1 \\
\hline
\end{tabular}

\begin{tabular}{|c|c|c|c|}
\hline$\theta^{3}$ & $a^{1}$ & $a^{2}$ & $a^{3}$ \\
\hline$a^{1}$ & -1 & -1 & 1 \\
\hline$a^{2}$ & -1 & -1 & 1 \\
\hline$a^{3}$ & -1 & 1 & 0 \\
\hline
\end{tabular}

Consider the following equilibrium: player one has the coarsest analogy partition and player two has the finest. Each player plays a pure separation strategy, each player's choice at state $\theta^{j}$ is $a^{j}$. The conjecture of player one is $\widehat{\sigma}_{2}^{1}\left(a^{j} \mid \theta^{k}\right)=$ $\frac{1}{3}$ for all $j, k=1,2,3$. Player two matches her action with the state and thus he is best-replying. Also, player one is best-replying since in state $\theta^{k}$ choosing $a_{k}$ gives expected payoff zero whereas other actions give negative expected payoffs given that player one expects two to choose each action with probability $\frac{1}{3}$. Thus, this is an ABEE but certainly not a Nash equilibrium, since player 1 is not choosing her best-response in a single state.

Furthermore, only outcomes $\left(a^{k}, a^{k}, \theta^{k}\right), k=1,2,3$ have a positive actual probability and each results with probability $\frac{1}{3}$. Thus, the sample distribution of player one's payoff assigns probability $\frac{1}{3}$ to payoffs $-1,0$ and 1 respectively. Since this is player one's expectation of payoffs given his equilibrium strategy, we have a PCABEE. Yet, neither is there for each $a^{k}$, a payoff $u^{k}$ such that for all $l$, for all $k, u_{1}\left(a^{l}, a^{k}, \theta^{l}\right)=u^{k}$, nor is there for each $\theta^{k}$, a payoff $u^{k}$ such that for all $l$, for all $k, u_{1}\left(a^{l}, a^{l}, \theta^{k}\right)=u^{k}$. Thus, we have a PCABEE even if neither condition (7) nor condition (8) in proposition (4) are satisfied.

The PCABEE in this example imposes restrictions on the prior distribution unlike proposition 4 , each state must have probability $1 / 3$. Therefore it is not similarly robust to changes in exogenous payoff uncertainty. More generally, only equilibria satisfying the condition in proposition 4 are robust to all perturbations of the prior of a given small extent.

The proposition illustrates that however small a perturbation will destabilize an equilibrium unless the condition in proposition 4 holds. This is due to the 
implied inconsistency between the actual and the expected payoff distribution. For the sake of simplicity, we consider only pure strategy strict equilibria. The equilibrium must be strict to avoid small perturbations affecting the optimality of the strategies themselves.

Proposition 5 Let $s^{*}$ be a pure strategy-profile of a PCABEE. Let the equilibrium be strict. For any $\varepsilon>0$, there exists a perturbation of the prior, $(1-\varepsilon) p+\varepsilon p^{o}$ where $p^{o} \neq p$, such that $s^{*}$ is not a strategy-profile of a PCABEE iff the conditions in proposition 4 are not satisfied.

Proof. In the appendix.

\section{$5 \quad$ Incorrect prior}

When opponents characteristics are observed after each round of play or when players can be assumed to have knowledge about exogenous uncertainty, it is plausible to assume the expectations about the prior to be eventually correct in a steady state of learning. Yet, in some contexts, there are outcomes of play where not all players get to observe the others' characteristics or types. This is the case for non-trading buyers in auctions or in bilateral trade where seller is better informed. In this section we relax the correct prior assumption and only require that the beliefs about others' characteristics are consistent with what one gets to observe.

With incorrect priors, there can be many more steady states than with correct priors. Despite this potential multiplicity, the payoff-confirming refinement reduces the number of consistent steady states sufficiently to allow clear cut implications on the set of equilibria even in rather general settings. Esponda (2008) considers monotone selection setups in which lower actions select lower distribution of quality (given strategies) and lower beliefs about quality induce players to choose lower actions. These setups comprise bilateral trade (Akerlof, 1970), for instance, and many applications where Eyster and Rabin's (2005) cursed equilibrium has been shown to alleviate selection problems and induce thicker markets. Esponda, quite surprisingly, shows that in games with monotone selection, markets are even thinner than in Nash equilibria when players learn the others' actions, their own payoffs, and occasionally the types of others, but fail to pay attention to correlations as in the cursed equilibrium. Indeed, our highly stylized bilateral trade example in section 2 illustrates Esponda's results: 
there is no selection problem in the Bayesian-Nash equilibrium of that particular game whereas a selection problem appears in a payoff-confirming steady state of learning. There is a number of applications where the aggravation of the selection problem takes place: in common-value auctions bidders bid less aggressively than in a Bayesian-Nash equilibrium, team work settings where team members put less effort in team work vis-à-vis a Bayesian-Nash equilibrium effort to mention a few. We urge an interested reader to see section 5 in Esponda (2008). Here our purpose is to show that Esponda's naive behavioral equilibrium can be considered as a special case of our payoff-confirming analogy-based expectation equilibrium with an incorrect prior.

Before discussing these issues, let us define more formally the primary class of games that Esponda studies. ${ }^{11}$ In the case of a "trade", each player's payoff only depends on the player's own action and a common value component, $v_{0}$, the exact value of which is typically unknown to at least one of the players. If the player does not trade, then payoff is zero. That the player trades is captured by the event $\left(a_{-i}, t_{0}\right) \in \Phi_{i}\left(\widehat{a}_{i}\right)$ to be determined shortly. Player $i$ 's payoff can now be written as

$$
u_{i}\left(a, t_{0}, v_{0}\right)=\left\{\begin{array}{cc}
\widetilde{u}_{i}\left(a_{i}, v_{0}\right) & \text { for }\left(a_{-i}, t_{0}\right) \in \Phi_{i}\left(\widehat{a}_{i}\right) \\
0 & \text { otherwise }
\end{array}\right.
$$

where $\theta_{0}=\left(t_{0}, v_{0}\right) \in T_{0} \times V_{0}$ represents payoff uncertainty and the vector $\theta_{1}, \ldots, \theta_{N} \in \Theta_{1} \times \ldots \times \Theta_{N}$ is the vector of types, one type for each of the players. Therefore, the type profile $\tilde{\theta}$ is a $N+2$-dimensional random variable. The following assumptions are made:

1. $\widetilde{t_{0}}$ is independent of $\widetilde{\theta}_{-0}$

2. $v_{0}$ and $\theta_{-0}$ are affiliated ${ }^{12}$

3. $\widetilde{u}_{i}$ is increasing in $v_{0}$

4. $\Phi_{i}\left(\widehat{a}_{i}\right)$ is non-decreasing in $\widehat{a}_{i}$ in the strong set order ${ }^{13}$ and in the inclusion set order.

5. $\widetilde{u}_{i}\left(a_{i}, v_{0}\right)$ is supermodular.

\footnotetext{
${ }^{11}$ The setup we use here is that of the on-line appendix of Esponda (2008) used to provide an example of a setup where the assumptions required in his main theorems hold.

${ }^{12} \theta$ are affiliated if for all $\theta^{\prime}$ and $\theta^{\prime \prime} \in \Theta, p\left(\theta^{\prime} \vee \theta^{\prime \prime}\right) p\left(\theta^{\prime} \wedge \theta^{\prime \prime}\right) \geq p\left(\theta^{\prime}\right) p\left(\theta^{\prime \prime}\right)$ where $\theta^{\prime} \vee \theta^{\prime \prime}=$ $\left(\max \left(\theta_{0}^{\prime}, \theta_{0}^{\prime \prime}\right), \ldots, \max \left(\theta_{N}^{\prime}, \theta_{N}^{\prime \prime}\right)\right)$ and $\theta^{\prime} \wedge \theta^{\prime \prime}=\left(\min \left(\theta_{0}^{\prime}, \theta_{0}^{\prime \prime}\right), \ldots, \min \left(\theta_{N}^{\prime}, \theta_{N}^{\prime \prime}\right)\right)$.

${ }^{13} \mathrm{~A}$ set $A \subset R$ is greater than a set $B \subset R$ in the strong set order if for any $a \in A$ and any $b \in B, \max (a, b)=a \vee b \in A$ and $\min (a, b)=a \wedge b \in B$.
} 
Denote by $\varphi_{i}\left(\sigma_{i}, \sigma_{-i}, \theta_{i}\right)$ the probability that $\left(a_{-i}, t_{0}\right) \in \Phi_{i}\left(\widehat{a}_{i}\right)$ given $\sigma_{i}, \sigma_{-i}$, and $\theta_{i}$, and let the subset $\theta_{-i}, \bar{\Theta}_{-i}\left(\sigma_{i}\left(\theta_{i}\right), \sigma_{-i}\left(\theta_{-i, 0} \mid \theta_{i}\right), \theta_{i}\right)$ of $\Theta_{-i}$ be defined as follows. It is the set of $\theta_{-i}$ which play actions resulting in $\left(a_{-i}, t_{0}\right) \in \Phi_{i}\left(\widehat{a}_{i}\right)$ with a positive probability given $\theta_{i}$ and $\sigma$.

Esponda illustrates the selection result by means of the concept of behavioral equilibrium. Proposition 6 establishes that the naive behavioral equilibrium corresponds to a PCABEE with an incorrect prior in the class of games satisfying (9) and assumptions 1 to 5. The proof and the definition of the behavioral equilibrium is relegated to the appendix.

Proposition 6 If for each $\theta_{i}$, the opponents' actions are observed and there is a positive probability $a_{i}$ and $a_{-i}$ such that $\varphi_{i}\left(a_{i}, a_{-i}, \theta_{i}\right)>0$, then every naive behavioral equilibrium is equivalent to a PCABEE with $\mathcal{A}_{i}=\left\{\left\{\bar{\Theta}_{-i}\left(\sigma_{i}, \theta_{i}, \widehat{\sigma}_{-i}^{i}\left(. \mid \theta_{i}\right)\right)\right\}_{\theta_{i} \in \Theta_{i}}\right\}$.

To build a correspondence between the naive behavioral equilibrium and the PCABEE, we need an analogy partition for each $i$ such that there is one class for each type of player $i$, the class of types of others from which this type "buys the product". Esponda's (2008) results can now be stated in terms of the PCABEE.

Proposition 7 (Esponda, 2008, theorem 1 and 2) For every payoff-confirming analogy-based best-reply, there is a Nash best-reply which is higher. The highest payoff-confirming ABEE is lower than the highest Bayesian-Nash equilibrium.

If $\theta$ are strictly affiliated, $\widetilde{u}_{i}\left(a_{i}, v_{0}\right)$ is strictly supermodular and $\Phi_{i}\left(\widehat{a}_{i}\right)$ is increasing (in the sense of 4 ), then

- every analogy-based best-reply is lower than any Nash-best-reply.

- the lowest ABEE is lower than the lowest Bayesian-Nash equilibrium.

The assumption that the prior is not known to the players is natural in many learning settings. Yet, the next proposition illustrates that it is important to distinguish between cases where prior is known and where it is not. The proposition shows that with a correct prior every PCABEE is a Bayesian-Nash equilibrium in Esponda's framework.

Proposition 8 Consider the class of games in section 5. Every PCABEE with a correct prior is a Bayesian Nash equilibrium. 


\section{Conclusion}

Jehiel and Koessler (2008) illustrate that rational players' optimal strategies can differ from a Bayesian-Nash equilibrium strategies when they base their conjectures on experience about others' behavior using stereotypical classifications. In this paper we consider such stereotypical learning assuming that players use their performance (payoffs) as a means to verify the consistency of the learned conjectures. We have identified conditions under which such Payoff-confirming analogy-based expectations equilibria can differ from Bayesian-Nash equilibria.

In a related paper, Esponda (2008) points out that payoff-information may have surprising consequences on steady states of learning if individuals are unable to detect correlations, as in the analogy-based expectation equilibria, and base their understanding of the uncertainty of the environment merely on their observations. ${ }^{14}$ Namely if players' conjecture on the prior distribution on types may be incorrect and correlation between types and strategies is not well understood, learning leads to an aggravation of adverse selection problems in common value environments. This paper complements the findings of Esponda by pointing out that the solution concept he uses can also be considered as a Payoffconfirming ABEE in an important class of games, namely in the prime class of games used by Esponda. Moreover, the current paper points out that nonBayesian Nash steady states exist even when conjecture on the prior is correct.

The payoff-confirming ABEE has perhaps three advantages when opposed with the naive Behavioral equilibrium: first, it appears simpler and more transparent of the two; second, it exists whenever the Bayesian Nash equilibrium does whereas the existence of a (naive) behavioral equilibrium is not guaranteed; third by considering the analogy-partition as a description of a player's stereotypes, it provides an interesting avenue for a game-theoretic analysis of these latter.

\section{$7 \quad$ Appendix}

\subsection{Proof of proposition 3}

Proof. If ABEE is BNE, then by Proposition 2 the ABEE is PCABEE.

On the other hand, if (5) or (6) holds for each $i$ such that $s_{i}\left(\theta^{m}\right) \neq s_{i}\left(\theta^{n}\right)$ and

\footnotetext{
${ }^{14}$ Esponda points out that mere existence of naive players of sufficient for the phenomenon - not every player needs to fail to account for correlations, i.e. to be naive.
} 
$\mathcal{A}_{j}=\left\{\left\{\theta^{1}, \theta^{2}\right\}\right\}$, then the ABEE is a PCABEE by lemma 1 below.

Consider now an ABEE which is a PCABEE and suppose to the contrary that the ABEE is not a BNE and there is $i$ such that $s_{i}\left(\theta^{m}\right) \neq s_{i}\left(\theta^{n}\right)$ and $\mathcal{A}_{j}=\left\{\left\{\theta^{1}, \theta^{2}\right\}\right\}$ and neither (5) nor (6) holds. Thus, by lemma 2, ABEE is not a PCABEE - a contradiction.

Lemma 1 Let $N=2, \Theta=\left\{\theta^{1}, \theta^{2}\right\}$ and $A_{i}=\left\{a_{i}^{1}, a_{i}^{2}\right\}$. Suppose that $s$ is a pure strategy profile of an ABEE. Let for each $i$ such that $s_{i}\left(\theta^{m}\right) \neq s_{i}\left(\theta^{n}\right)$ and $\mathcal{A}_{j}=\left\{\left\{\theta^{1}, \theta^{2}\right\}\right\}$ either (5) or (6) hold.

Then the ABEE is PCABEE.

Proof. Define the probability of $j$ getting payoff $u$ given strategy profile $\sigma$ as $g_{\sigma}^{j}(u)=\sum_{\left\{a, \theta \mid u=u_{j}(a, \theta)\right\}} p(\theta) \sigma_{i}\left(a_{i} \mid \theta\right) \sigma_{j}\left(a_{j} \mid \theta\right)$.

Let there be $i$ such that $s_{i}\left(\theta^{m}\right) \neq s_{i}\left(\theta^{n}\right)$ and $\mathcal{A}_{j}=\left\{\left\{\theta^{1}, \theta^{2}\right\}\right\}$. There are three subcases to consider: first

$$
\begin{aligned}
u_{j}\left(s_{j}\left(\theta^{m}\right), r_{i}\left(\theta^{m}\right) ; \theta^{m}\right) & =u_{j}\left(s_{j}\left(\theta^{m}\right), s_{i}\left(\theta^{m}\right) ; \theta^{m}\right) \\
& =u_{j}\left(s_{j}\left(\theta^{n}\right), r_{i}\left(\theta^{n}\right) ; \theta^{n}\right) \\
& \left.=u_{j}\left(s_{j}\left(\theta^{n}\right), s_{i}\left(\theta^{n}\right) ; \theta^{n}\right)\right)
\end{aligned}
$$

in which case both conditions hold. In this first subcase trivially $g_{\left(s_{j}, \widehat{\sigma}_{i}^{j}\right)}^{j}\left(u_{j}\left(s_{j}\left(\theta^{m}\right), s_{i}\left(\theta^{m}\right) ; \theta^{m}\right)\right)=1=g_{s}^{j}\left(u_{j}\left(s_{j}\left(\theta^{m}\right), s_{i}\left(\theta^{m}\right) ; \theta^{m}\right)\right)$. Thus, $s$ is a payoff confirming analogy-based expectation equilibrium.

In the second case only (6) holds but not (5). For each $\theta^{m}$, the perceived probability that $u_{j}\left(s_{j}\left(\theta^{m}\right), s_{i}\left(\theta^{m}\right) ; \theta^{m}\right)$ results is

$$
\begin{aligned}
g_{\left(s_{j}, \widehat{\sigma}_{i}^{j}\right)}^{j}\left(u_{j}\left(s_{j}\left(\theta^{m}\right), s_{i}\left(\theta^{m}\right) ; \theta^{m}\right)\right) & =\left[f\left(\theta^{m}\right)\right] \beta^{j}\left(s_{i}\left(\theta^{m}\right)\right)+\left[1-f\left(\theta^{m}\right)\right] \beta^{j}\left(s_{i}\left(\theta^{m}\right)\right) \\
& =\left[f\left(\theta^{m}\right)\right]^{2}+\left[1-f\left(\theta^{m}\right)\right] f\left(\theta^{m}\right) \\
& =f\left(\theta^{m}\right) \\
& =g_{s}^{j}\left(u_{j}\left(s_{j}\left(\theta^{m}\right), s_{i}\left(\theta^{m}\right) ; \theta^{m}\right)\right) .
\end{aligned}
$$

Thus, $s$ is a payoff confirming analogy-based expectation equilibrium.

Third, if only (5) holds and not (6), we have that

$$
\begin{aligned}
g_{\left(s_{j}, \widehat{\sigma}_{i}^{j}\right)}^{j}\left(u_{j}\left(s_{j}\left(\theta^{n}\right), s_{i}\left(\theta^{n}\right) ; \theta^{n}\right)\right) & =\left[f\left(\theta^{n}\right)\right]\left[\beta^{j}\left(s_{i}\left(\theta^{n}\right)\right)+\beta^{j}\left(r_{i}\left(\theta^{n}\right)\right)\right] \\
& =\left[f\left(\theta^{n}\right)\right] \\
& =g_{s}^{j}\left(u_{j}\left(s_{j}\left(\theta^{n}\right), s_{i}\left(\theta^{n}\right) ; \theta^{n}\right)\right) .
\end{aligned}
$$


Thus, $s$ is a payoff confirming analogy-based expectation equilibrium.

Lemma 2 Let $N=2, \Theta=\left\{\theta^{1}, \theta^{2}\right\}$ and $A_{i}=\left\{a_{i}^{1}, a_{i}^{2}\right\}$. Suppose that $s$ is a pure strategy profile of an $A B E E$.

If there is $i$ such that $s_{i}\left(\theta^{m}\right) \neq s_{i}\left(\theta^{n}\right)$ and $\mathcal{A}_{j}=\left\{\left\{\theta^{1}, \theta^{2}\right\}\right\}$ and neither

$$
\text { for all } m, u_{j}\left(s_{j}\left(\theta^{m}\right), r_{i}\left(\theta^{m}\right) ; \theta^{m}\right)=u_{j}\left(s_{j}\left(\theta^{m}\right), s_{i}\left(\theta^{m}\right) ; \theta^{m}\right)
$$

nor

$$
\text { for all } \left.m, u_{j}\left(s_{j}\left(\theta^{m}\right), r_{i}\left(\theta^{m}\right) ; \theta^{m}\right)=u_{j}\left(s_{j}\left(\theta^{n}\right), s_{i}\left(\theta^{n}\right) ; \theta^{n}\right)\right)
$$

where $r_{i}\left(\theta^{m}\right)$ is the action not chosen by $i$ at $\theta^{m}$,

then the $A B E E$ is not a PCABEE.

\section{Proof.}

We use proof by contradiction. There are two subcases to consider. Suppose first, that there is $m$ such that

$$
\left.u_{j}\left(s_{j}\left(\theta^{m}\right), r_{i}\left(\theta^{m}\right) ; \theta^{m}\right) \notin\left\{u_{j}\left(s_{j}\left(\theta^{m}\right), s_{i}\left(\theta^{m}\right) ; \theta^{m}\right), u_{j}\left(s_{j}\left(\theta^{n}\right), s_{i}\left(\theta^{n}\right) ; \theta^{n}\right)\right)\right\} .
$$

Define the probability of $j$ getting payoff $u$ given strategy profile $\sigma$ as

$$
g_{\sigma}^{j}(u)=\sum_{\left\{a, \theta \mid u=u_{j}(a, \theta)\right\}} p(\theta) \sigma_{i}\left(a_{i} \mid \theta\right) \sigma_{j}\left(a_{j} \mid \theta\right) .
$$

Since $s_{i}\left(\theta^{m}\right) \neq s_{i}\left(\theta^{n}\right), j$ expects $u_{j}\left(s_{j}\left(\theta^{m}\right), r_{i}\left(\theta^{m}\right) ; \theta^{m}\right)$ to result with a positive probability,

$$
g_{s_{j}, \widehat{\sigma}_{i}^{j}}^{j}\left(u_{j}\left(s_{j}\left(\theta^{m}\right), r_{i}\left(\theta^{m}\right) ; \theta^{m}\right)\right)>0
$$

But since (10) holds, $g_{s_{j}, \sigma_{i}}^{j}\left(u_{j}\left(s_{j}\left(\theta^{m}\right), r_{i}\left(\theta^{m}\right) ; \theta^{m}\right)\right)=0$ which contradicts the consistency condition of PCABEE and thus the ABEE is not PCABEE.

In the second subcase, suppose in addition to $s_{i}\left(\theta^{m}\right) \neq s_{i}\left(\theta^{n}\right)$ that there is $m$ and $i$ such that

$$
\begin{aligned}
u_{j}\left(s_{j}\left(\theta^{m}\right), r_{i}\left(\theta^{m}\right) ; \theta^{m}\right) & =u_{j}\left(s_{j}\left(\theta^{m}\right), s_{i}\left(\theta^{m}\right) ; \theta^{m}\right) \\
& \left.=u_{j}\left(s_{j}\left(\theta^{n}\right), r_{i}\left(\theta^{n}\right) ; \theta^{n}\right)\right) \\
& \left.\neq u_{j}\left(s_{j}\left(\theta^{n}\right), s_{i}\left(\theta^{n}\right) ; \theta^{n}\right)\right)
\end{aligned}
$$


Then

$$
\begin{aligned}
g_{\left(s_{j}, \widehat{\sigma}_{i}^{j}\right)}^{j}\left(u_{j}\left(s_{j}\left(\theta^{m}\right), s_{i}\left(\theta^{m}\right) ; \theta^{m}\right)\right) & =f\left(\theta^{m}\right)+f\left(\theta^{n}\right) \beta^{j}\left(r_{i}\left(\theta^{n}\right)\right) \\
& \neq f\left(\theta^{m}\right) \\
& =g_{s}^{j}\left(u_{j}\left(s_{j}\left(\theta^{m}\right), s_{i}\left(\theta^{m}\right) ; \theta^{m}\right)\right)
\end{aligned}
$$

and thus ABEE is not PCABEE.

\subsection{Proof of proposition 4}

Proof of proposition 4. Let for each $j$ and $\alpha_{j}$ such that $\sigma_{-j}\left(\theta^{m}\right) \neq$ $\sigma_{-j}\left(\theta^{n}\right)$ and $\theta^{m}, \theta^{n} \in \alpha_{j}$, for all $\theta \in \alpha_{j}$, for all action profiles of players other than $j, a_{-j}^{*}=\left(a_{1}^{*}, \ldots, a_{j-1}^{*}, a_{j+1}^{*}, \ldots, a_{N}^{*}\right)$ such that for each $i \neq j$ there is $\theta^{\prime} \in \alpha_{j}$ such that $a_{i}^{*} \in \operatorname{supp} \sigma_{i}\left(\theta^{\prime}\right)$ for all $a_{j} \in \operatorname{supp} \sigma_{j}(\theta)$,

$$
u_{j}\left(a_{j}, a_{-j} ; \theta\right)=u_{\theta}
$$

Now,

$$
\begin{aligned}
& \sum_{\left\{a, \theta \mid u_{j}=u_{j}(a, \theta)\right\}} p(\theta) \widehat{\sigma}_{-j}\left(a_{-j} \mid \theta_{-j}\right) \sigma_{j}\left(a_{j} \mid \theta_{j}\right) \\
= & \sum_{\alpha_{j} \in \mathcal{A}_{j}} \sum_{\left\{\left(a_{j}, \theta\right) \mid u_{j}=u_{j}(a, \theta), \theta \in \alpha_{i}\right\}} p(\theta) \widehat{\sigma}_{-j}\left(a_{-j} \mid \theta_{-j}\right) \sigma_{j}\left(a_{j} \mid \theta_{j}\right) \\
= & \sum_{\alpha_{j} \in \mathcal{A}_{j}} \sum_{\theta^{n} \in \alpha_{j}} p\left(\theta^{n}\right) \sigma_{j}\left(a_{j} \mid \theta_{j}^{n}\right) \sum_{a_{-j}^{*}} \widehat{\sigma}_{-j}\left(a_{-j}^{*} \mid \theta_{-j}^{n}\right) \\
= & \sum_{\alpha_{j} \in \mathcal{A}_{j}} \sum_{\theta^{n} \in \alpha_{j}} p\left(\theta^{n}\right) \sigma_{j}\left(a_{j} \mid \theta_{j}^{n}\right) \\
= & \sum_{\alpha_{j} \in \mathcal{A}_{j}} \sum_{\theta^{n} \in \alpha_{j}} p\left(\theta^{n}\right) \sigma_{j}\left(a_{j} \mid \theta_{j}^{n}\right) \sum_{a_{-j}} \sigma_{-j}\left(a_{-j} \mid \theta_{j}\right) \\
= & \sum_{\alpha_{j} \in \mathcal{A}_{j}} p(\theta) \sigma_{-j}\left(a_{-i} \mid \theta_{-j}\right) \sigma_{j}\left(a_{j} \mid \theta_{j}\right) \\
= & \left.\sum_{\left\{a, \theta \mid u_{i}=u_{i}(a, \theta), \theta \in \alpha_{j}\right\}} p(\theta) \sigma_{-j}(a, \theta)\right\}
\end{aligned}
$$

where the second equality follows from the fact that, in an analogy class, for a state in the class and for an action that is chosen with a positive probability by $j$ in that state the payoff is the same for any action profile of players other than $j$ to which $\widehat{\sigma}_{-j}$ assigns a positive probability. The third and the fourth 
equality follow because a conjecture and a strategy is a probability distribution and thus sums up to one. $\left(\sum_{a_{-j}^{*}} \widehat{\sigma}_{-j}\left(a_{-j} \mid \theta_{-j}\right)=1=\sum_{a_{-j}} \sigma_{-j}\left(a_{-j} \mid \theta_{j}\right)\right)$ and only actions which are assigned a positive probability in the average strategy of the analogy class can be assigned a positive probability in the actual strategy.

Let for each $j$ and $\alpha_{j}$ such that $\sigma_{-j}\left(\theta^{m}\right) \neq \sigma_{-j}\left(\theta^{n}\right)$ and $\theta^{m}, \theta^{n} \in \alpha_{j}$, for all action profiles of players other than $j, a_{-j}^{*}=\left(a_{1}^{*}, \ldots, a_{j-1}^{*}, a_{j+1}^{*}, \ldots, a_{N}^{*}\right)$ such that for each $i \neq j$ there is $\theta^{\prime} \in \alpha_{j}$ such that $a_{i}^{*} \in \operatorname{supp} \sigma_{i}\left(\theta^{\prime}\right)$, for all $\theta \in \alpha_{j}$ and $a_{j} \in \operatorname{supp} \sigma_{j}(\theta)$

$$
u_{j}\left(a_{j}, a_{-j} ; \theta\right)=u_{a_{-j}}
$$

Now,

$$
\begin{aligned}
& \sum_{\left\{a, \theta \mid u_{j}=u_{j}(a, \theta)\right\}} p(\theta) \widehat{\sigma}_{-j}\left(a_{-j} \mid \theta_{j}\right) \sigma_{j}\left(a_{j} \mid \theta_{j}\right) \\
= & \sum_{\alpha \in \mathcal{A}_{j}} \sum_{\left\{(a, \theta) \mid u_{j}=u_{j}(a, \theta), \theta \in \alpha\right\}} p(\theta) \sigma_{j}\left(a_{j} \mid \theta_{j}\right) \widehat{\sigma}_{-j}\left(a_{-j} \mid \theta\right) \\
= & \sum_{\alpha \in \mathcal{A}_{j}} \sum_{a_{-j}^{*}} \widehat{\sigma}_{-j}\left(a_{-j}^{*} \mid \alpha\right) \sum_{\theta \in \alpha} p(\theta) \sigma_{j}\left(a_{j} \mid \theta_{j}\right) \\
= & \sum_{\alpha \in \mathcal{A}_{j}} \sum_{\theta \in \alpha} p(\theta) \sigma_{j}\left(a_{j} \mid \theta_{j}\right) \\
= & \sum_{\alpha \in \mathcal{A}_{j}\left\{\left(a_{j}, \theta\right) \mid u_{j}=u_{j}(a, \theta), \theta \in \alpha\right\}} p(\theta) \sigma_{j}\left(a_{j} \mid \theta_{j}\right) \sum_{a_{-j}} \sigma_{-j}\left(a_{-j} \mid \theta_{-j}\right) \\
= & \sum_{\alpha \in \mathcal{A}_{j}} \sum_{\left\{(a, \theta) \mid u_{j}=u_{j}(a, \theta), \theta \in \alpha\right\}} p(\theta) \sigma_{j}\left(a_{j} \mid \theta_{j}\right) \sigma_{-j}\left(a_{-j} \mid \theta_{-j}\right) \\
= & \sum_{\left\{a, \theta \mid u_{i}=u_{i}(a, \theta)\right\}} p(\theta) \sigma_{-j}\left(a_{-i} \mid \theta_{-j}\right) \sigma_{j}\left(a_{j} \mid \theta_{j}\right)
\end{aligned}
$$

where the second equality follows from the fact that for a given action profile $a_{-j}^{*}$ in supp $\widehat{\sigma}_{-j}(\alpha)$ the payoff $u_{j}\left(a_{j}, a_{-j}^{*}, \theta\right)$ is the same for each $\theta$ in $\alpha_{j}$ and $\left(a_{j}, \theta\right)$ such that $a_{j} \in \operatorname{supp} \sigma_{j}(\theta)$. The third and the fourth equality follow because a strategy and a conjecture are probability distributions and only actions which are assigned a positive probability in the average strategy of the analogy class can be assigned a positive probability in the actual strategy.

\subsection{Proof of proposition 5}

Proof. Consider an arbitrary player $i$ and an arbitrary analogy class of that player. Let $K$ be the number of states in the analogy class. Let $s_{-i}^{l}$ be the 
action profile of others chosen at state $l$. Let us construct a square matrix $U:=\left[u\left(s_{i}^{*}\left(\theta_{i}^{k}\right), s_{-i}^{l}, \theta^{k}\right)\right]_{k, l=1}^{K}$. The payoff-confirming condition can be written as a system of $\#\left\{u_{i}\left(s_{i}^{*}\left(\theta_{i}^{k}\right), s_{-i}^{*}\left(\theta^{k}\right), \theta^{k}\right) \mid k=1, \ldots, K\right\}$ equations, that is, the number of actual generic payoffs which cannot be higher than $\mathrm{K}$ by construction. Define $f^{u}(p)$ as the difference in the expected and the actual probability of payoff $\mathrm{u}$ given $\mathrm{p}$. An equation of the system for a given generic payoff $u$ reads

$$
\begin{aligned}
f^{u}(p) & =\sum_{k=1}^{K} p_{k} \sum_{l=1}^{K} \widehat{\sigma}_{-i}^{i}\left(s_{-i}^{l} \mid \theta^{k}\right) \mathcal{I}\left(U_{l k}=u\right)-\sum_{k} p_{k} \mathcal{I}\left(U_{k k}=u\right)=0 \\
& \Leftrightarrow \sum_{k=1}^{K} p_{k} \sum_{l=1}^{K} \frac{\sum_{m} p_{m} \mathcal{I}\left(s_{-i}^{m}=s_{-i}^{l}\right)}{\sum_{m} p_{m}} \mathcal{I}\left(U_{l k}=u\right)-\sum_{k} p_{k} \mathcal{I}\left(U_{k k}=u\right)=0
\end{aligned}
$$

where $\mathcal{I}\left(U_{l k}=u\right)$ is the indicator that the element at the $l$ th row and $k$ th column of $U$ satisfies $U_{l k}=u$. The first term of the difference is the probability by which the player expects payoff $u$ and the second term is the actual probability of payoff $u$. The condition says merely that these probabilities must coincide.

The effect of a change of the prior probability $p_{\kappa}$ of a given state $\theta^{\kappa}$ on $f^{u}(p)$ is given by

$$
\begin{aligned}
f_{\kappa}^{u}(p) & =\sum_{l} \widehat{\sigma}_{-i}^{i}\left(s_{-i}^{l} \mid \theta^{\kappa}\right) \mathcal{I}\left(U_{l \kappa}=u\right)-\mathcal{I}\left(U_{\kappa \kappa}=u\right) \\
& +\sum_{k} p_{k} \frac{\partial \sum_{l} \widehat{\sigma}_{-i}^{i}\left(s_{-i}^{l} \mid \theta^{k}\right)}{\partial p_{\kappa}} \mathcal{I}\left(U_{l k}=u\right) \\
& =\sum_{l} \frac{\sum_{m} p_{m} \mathcal{I}\left(s_{-i}^{m}=s_{-i}^{l}\right)}{\sum_{k} p_{k}} \mathcal{I}\left(U_{l \kappa}=u\right)-\mathcal{I}\left(U_{\kappa \kappa}=u\right) \\
+ & \sum_{k} p_{k} \sum_{l} \mathcal{I}\left(U_{l k}=u\right)\left(\frac{1}{\sum_{m} p_{m}}-\frac{\sum_{m} p_{m} \mathcal{I}\left(s_{-i}^{m}=s_{-i}^{l}\right)}{\left(\sum_{m} p_{m}\right)^{2}}\right)
\end{aligned}
$$

Suppose that the condition in proposition 4 is violated. Then there must be two different states $k \neq \kappa$ and actions $l \neq \lambda$ chosen with a positive probability in the analogy class such that $U_{k l}=U_{\kappa \lambda}$.

Consider a perturbation of the prior probabilities of two states, $k$ and $\kappa$ only, so that $\Delta p_{k}=-\Delta p_{\kappa}$. The prior probabilities of the other states remain unaltered. Thus, the initial strategies do not constitute a PCABEE of the 
perturbed game if $-f_{k}^{u}(p) \Delta p_{k} \neq f_{\kappa}^{u}(p) \Delta p_{\kappa}$ or

$$
-\frac{\Delta p_{k}}{\Delta p_{\kappa}} \neq \frac{f_{\kappa}^{u}(p)}{f_{k}^{u}(p)}
$$

where by construction $f_{k}^{u}(p), f_{\kappa}^{u}(p) \neq 0$. Thus, almost all such perturbations destabilize the original PCABEE and such perturbations exist for any $\varepsilon>0$.

The converse holds by proposition 4 .

\subsection{Behavioral equilibrium and PCABEE}

Assumptions on player's conjectures and how they handle information in a behavioral equilibrium are the following:

Definition 4 (Behavioral equilibrium ${ }^{15}$ )

B1 Players believe that $\widetilde{t_{0}}$ is independent of $\left(\widetilde{\theta}, \widetilde{a}_{-i}\right)$.

B2 Every player has correct conjectures about the probability of $\left(\widetilde{t}_{0}, \widetilde{a}_{-i}\right) \in$ $\Phi_{i}(\widehat{a})$ (and therefore about $\left.\left(\widetilde{t_{0}}, \widetilde{a}_{-i}\right) \notin \Phi_{i}(\widehat{a})\right)$ given $\theta_{i}$.

B3 Payoffs are observed.

B4 Players suppose that the opponents' actions are independent of the opponents' types.

\section{Proof of proposition 6 .}

We will show that the properties of the behavioral equilibrium, B1-B4, in the given context, (9) and properties 1 to 4 , will imply certain conjectures. We then show that there is an analogy partition and a PCABEE with an incorrect prior the conjecture-restrictions of which are satisfied by these conjectures.

Given strategies $\sigma$, B4 implies the following conjectures on player strategies: type $\theta_{i}$ of player $i$ conjectures that every opponent type profile that she conjectures to have a positive probability plays the marginal distribution of actions conditional on $\theta_{i}$

$$
\widehat{\sigma}_{-i}^{i}\left(a_{-i} \mid \theta_{i}\right)=\sum_{\theta_{-i} \in \Theta_{-i}} \sigma_{-i, 0}\left(a_{-i} \mid \theta_{-i, 0}\right) p\left(\theta_{-i} \mid \theta_{i}\right) .
$$

\footnotetext{
${ }^{15}$ This corresponds to Esponda's naive behavioral equilibrium.
} 
B1 implies that type $\theta_{i}$ of player $i$ conjectures that each type profile, $\theta_{-i}$, given $\theta_{i}$ has a probability

$$
\widehat{\mu}^{i}\left(\theta_{-i} \mid \theta_{i}\right)=\widehat{\mu}^{i}\left(v_{0}, \theta_{-i, 0} \mid \theta_{i}\right) \widehat{\mu}^{i}\left(t_{0} \mid \theta_{i}\right) .
$$

B4 and the fact that actions are observed implies that conjectures about strategies satisfy (11). B1, B2, that $a_{-i}$ are observed for each $\theta_{i}$ and the fact that $\varphi_{i}\left(\sigma_{i}, \sigma_{-i}, \theta_{i}\right)$ depends only on $\left(a_{-i}, t_{0}\right)$ for each $\sigma_{i}$ implies that the marginal conjecture about $t_{0}$ must be correct. If $i$ had an incorrect conjecture about the distribution of $t_{0}$, she would necessarily have an incorrect probability estimate about $\varphi_{i}\left(\sigma_{i}, \sigma_{-i}, \theta_{i}\right)$. Thus,

$$
\widehat{\mu}^{i}\left(t_{0} \mid \theta_{i}\right)=\sum_{v_{0}, \theta_{-i} \in V_{0} \times \Theta_{-i, 0}} p\left(v_{0}, \theta_{-i, 0}, t_{0} \mid \theta_{i}\right) .
$$

Given $\theta_{i}$, by assumption $\varphi_{i}\left(\sigma_{i}, \sigma_{-i}, \theta_{i}\right)>0$ and therefore, by B2, $\varphi_{i}\left(\sigma_{i}, \sigma_{-i}, \theta_{i}\right)=\varphi_{i}\left(\sigma_{i}\left(\theta_{i}\right), \widehat{\sigma}_{-i}^{i}\left(. \mid \theta_{i}\right), \theta_{i}\right)>$ 0 . B3 and the fact that $u_{i}$ is increasing in $v_{0}$ implies that player $i$ observes the realization of $v_{0}$ iff there is $a_{i} \quad \in \operatorname{supp}\left\{\sigma_{i}\right\} \quad$ and $a_{-i} \in \operatorname{supp}\left(\widehat{\sigma}_{-i}^{i}\left(. \mid \theta_{i}\right)\right)$ s.t. $\left(a_{-i}, t_{0}\right) \in \Phi_{i}(\widehat{a})$. Necessary for the equilibrium conjectures about the strategies is also that all observed $v_{0}$ are expected with a positive probability. Since by B4 actions are non-correlated with types, every expected type must play the same strategy: the strategy described in (11). Moreover, it must be the case that no $v_{0}^{\prime}$ with a zero actual probability (i.e. there is no type profile in $\bar{\Theta}\left(\sigma_{i}\left(\theta_{i}\right), \widehat{\sigma}_{-i}^{i}\left(. \mid \theta_{i}\right), \theta_{i}\right)$ with $v_{0}^{\prime}$ as its first scalar), is conjectured to have a positive probability, since, first, whether a given $v_{0}$ is observed is independent of $t_{0}$ and $a_{-i}$, second, every expected $v_{0}$ must play the same strategy and, third, $\varphi_{i}\left(\sigma_{i}, \widehat{\sigma}_{-i}^{i}\left(. \mid \theta_{i}\right), \theta_{i}\right)>0$. Otherwise, that $v_{0}$ would be conjectured to be observed which would conflict the consistency of the conjecture. Since by B3 payoffs are observed and $\widetilde{u}_{i}$ is increasing in $v_{0}$ player $i$ can deduce $v_{0}$ conditional on $\left(a_{-i}, t_{0}\right) \in \Phi_{i}\left(\widehat{a}_{i}\right)$. Therefore $\widehat{\mu}^{i}\left(v_{0}, \theta_{-i, 0} \mid \theta_{i}\right)$ must satisfy 


$$
\begin{gathered}
\sum_{\substack{\theta_{1}, \ldots, \theta_{i-1}, \theta_{i+1}, \ldots, \theta_{n} \in \\
\Theta_{1}, \ldots, \Theta_{i-1}, \Theta_{i+1}, \ldots \Theta_{n}}} \widehat{\mu}^{i}\left(v_{0}, \theta_{1}, \ldots, \theta_{i-1}, \theta_{i+1}, \ldots, \theta_{n} \mid \theta_{i}\right) \\
=\frac{1}{\varphi_{i}\left(\sigma_{i}\left(\theta_{i}\right), \sigma_{-i}\left(\theta_{-i} \mid \theta_{i}\right), \theta_{i}\right)} \times \\
\sum_{\substack{\left\{\left(a_{i}, a_{-i}, t_{0}, \theta_{-i, 0}\right) \mid \\
\left(a_{-i}, t\right) \in \Phi_{i}\left(\hat{a}_{i}\right)\right\}}}\left(\sigma_{i}\left(a_{i} \mid \theta_{i}\right) \sigma_{-i}\left(a_{-i} \mid \theta_{-i}\right) \times\right. \\
\left.\quad f\left(v_{0}, \theta_{1}, \ldots, \theta_{n}, t_{0} \mid \theta_{i}\right)\right) .
\end{gathered}
$$

The expected marginal distribution of $v_{0}$ given $\theta_{i}$ coincides with the observed marginal distribution of $v_{0}$ conditional on there being $t_{0}, v_{0}, \theta_{-i, 0}$, belonging to $\bar{\Theta}_{-i}\left(\sigma_{i}\left(\theta_{i}\right), \hat{\sigma}_{-i}^{i}\left(. \mid \theta_{i}\right), \theta_{i}\right)$.

It is easy to see that given a unique analogy class $\alpha\left(\theta_{i}\right)=\bar{\Theta}_{-i}\left(\sigma_{i}\left(\theta_{i}\right), \widehat{\sigma}_{-i}^{i}\left(. \mid \theta_{i}\right)\right.$, $\theta_{i}$ ) for each $\theta_{i}$, equations (11) and (12) impose the required average-strategy and payoff-confirming restrictions, (4), respectively, on the corresponding payoffconfirming ABEE. Moreover $\bar{\Theta}_{-i}\left(\sigma_{i}\left(\theta_{i}\right), \widehat{\sigma}_{-i}^{i}\left(. \mid \theta_{i}\right), \theta_{i}\right)$ is a subset of $\Theta$, as a unique analogy class it is distinct from all other, and the conjectured probability of $\bar{\Theta}_{-i}\left(\sigma_{i}\left(\theta_{i}\right), \widehat{\sigma}_{-i}^{i}\left(. \mid \theta_{i}\right), \theta_{i}\right)$ coincides with its actual probability. Therefore, for each $\mathrm{BE}$ we have established a corresponding PCABEE with an incorrect prior.

Proof of proposition 8. (Sketch). Given $\theta_{i}$, we suppose for simplicity that the analogy partitions are over $\Theta_{1}, \ldots, \Theta_{i-1}, \Theta_{i+1}, \ldots, \Theta_{n}$.

By the definition of the ABEE, every type in each class plays the average strategy of the class. For all $i$, the strategies must be non-decreasing in $\theta_{i}$ since there is a positive affiliation between $v_{0}$ and $\theta_{i}$ and the payoff function $u_{i}$ is supermodular in $v_{0}$ and $a_{i}$. Thus, if not all types in the class play the same strategy, there exists a threshold type in the class such that types greater than this type are believed to choose lower actions than they actually are and types below the threshold action are believed to choose higher actions than they actually are. But by assumption 4 and by assumption 1 and since all types are expected to use the same strategy, all types are expected to be observed equally easily. Moreover, the players have a correct prior. Putting these two together, in each class where some type of the class puts a positive probability on an action $a_{-i}$ such that $\left(a_{-i}, t_{0}\right) \in \Phi_{i}\left(a_{i}^{*}\right)$ (call these analogy classes visible), the 
expected distribution of types conditional on $\left(a_{-i}, t_{0}\right) \in \Phi_{i}\left(a_{i}^{*}\right)$ coincides with the prior conditional on the analogy class.

But this can only be so if (i) all types actually play the same strategies or if (ii) the set-orders are non-increasing for the actions which have a positive probability in the class (given $i^{\prime}$ s equilibrium strategy). In the first case, the beliefs about the strategies in this analogy class must be correct. In the second, the incorrect beliefs are not relevant for best-replying. Thus, WLOG, one can assume that beliefs are correct in every analogy class where there is a type who chooses an action $a_{-i}$ such that $\left(a_{-i}, t_{0}\right) \in \Phi_{i}\left(a_{i}^{*}\right)$ results with a positive probability.

Now consider analogy classes where there is no type who chooses an action $a_{-i}$ such that there is $t_{0}$ and $a_{i}^{*}\left(a_{i}^{*}\right.$ such that $i$, chooses it with a positive probability given that opponent type) such that $\left(a_{-i}, t_{0}\right) \in \Phi_{i}\left(a_{i}^{*}\right)$ results with a positive probability. (Call such analogy classes invisible.) By, (9), payoff zero is expected and payoff zero also results. Moreover, due to monotonicities, we can partition types (or analogy classes) into invisible types who all choose so that $\left(a_{-i}, t_{0}\right) \notin \Phi_{i}\left(a_{i}^{*}\right)$ and visible types who all choose so that $\left(a_{-i}, t_{0}\right) \in \Phi_{i}\left(a_{i}^{*}\right)$.

Suppose that $i$ 's strategy is not a Nash best reply. By our findings above, this must be due to the incorrect beliefs about the behavior in the invisible analogy classes. By Proposition 7, we know that all players' best-replies are below the Nash best-replies. Suppose that there is player $i$ for whom this holds strictly.

Let us begin with a BNE with correct beliefs. Let us show first that other choosing strategies which first-order stochastically dominate the BNE-strategies imply an incentive for $i$ to increase her strategy above the BNE-strategy. Let us then show that introducing coarser analogy classification also implies an incentive to increase one's strategy. These two forces imply a contradiction with the finding in Proposition 7.

Suppose first that the actions of others are now reduced to any actions weakly lower than their Nash best replies and that $i^{\prime}$ s conjectures correctly capture this change. By assumption $5, i$ has an incentive to choose a weakly higher action than her Nash best-reply.

Now moreover, introduce an analogy partition of types of $-i$ for $i$. Let this analogy partition be finer (as required) than the partioning into visible and invisible types induced by $i$ 's and $-i$ 's strategies and $\left(a_{-i}, t_{0}\right) \in \Phi_{i}\left(a_{i}^{*}\right)$. Given that $i$ 's beliefs about types in the visible partition must be correct, introducing analogy classes to visible types leaves incentives unchanged. Introducing analogy 
partitions into invisible types induces expectations where in each analogy class low types are expected to choose higher $a_{-i}$ than they did with the correct expectations. Moreover, high types in each class are expected to choose lower actions. Yet, the probability of each class must be expected correctly. Thus, higher opponent types and thus higher $v_{0}$ are expected with a higher probability. Thus, this also increases $i$ 's incentive to choose higher $a_{i}$ due to assumption 5 . Thus, we have a contradiction.

\section{References}

[1] Arrow, K. (1998): What Has Economics to Say about Racial Discrimination? J. Econ. Persp., 91-100.

[2] Battigalli, P. (1987): Comportamento razionale ed equilibrio nei giochi e nelle situazioni sociali. Unpublished dissertation. Università Bocconi. Milan.

[3] Battigalli, P.; Gilli, M.; Molinari C. (1992): Learning and Convergence to Equilibrium in Repeated Strategic Interactions: An Introductory Survey. Ricerche Economiche, 46, 335-378.

[4] Battigalli, P.; Guaitoli, D. (1997): Conjectural Equilibrium and Rationalizability in a Game with Incomplete Information. In Decisions, Games and Markets. Kluwer Academic Publishers. Dodrecht.

[5] Dekel, E.; Fudenberg, D.; Levine, D.K (2004): Learning to Play Bayesian Games. Games Econ. Behav. 46, 282-303.

[6] Esponda, I. (2008): Behavioral Equilibrium in Economies with Adverse Selection. Forthcoming, Amer. Econ. Rev.

[7] Eyster, E.; Rabin, M. (2005): Cursed Equilibrium. Econometrica 73, 16231672.

[8] Fryer, R. and Jackson, M. O. (2008): A Categorical Model of Cognition and Biased Decision Making. B.E. Journal of Theoretical Economics (Contributions) 8, (1) , Article 6. DOI: 10.2202/1935-1704.1357.

[9] Fudenberg, D., Kreps, D.M. (1995). Learning in Extensive Games, I: SelfConfirming Equilibria. Games Econ. Behav. 8, 20-55 
[10] Fudenberg, D.; Levine D.K. (1993): Self-confirming equilibrium. Econometrica $61,523-545$.

[11] Fudenberg, D.; Levine D.K. (1998): The Theory of Learning in Games. MIT Press. Cambridge, MA.

[12] Jehiel, P. (2005): Analogy-Based Expectation Equilibrium. J. Econ. Theory, 123, 81-104.

[13] Jehiel, P.; Koessler, F. (2008): Revisiting Games of Incomplete Information with Analogy-based Expectations. Games Econ. Behav. 62, 533-557.

[14] Loury, G. (1998): Discrimination in the Post-Civil Rights Era: Beyond Market Interactions. J. Econ. Persp., 12, 117-126 .

[15] Mailath, G.; Samuelson, L.; Shaked, A. (2000): Endogenous Inequality in Integrated Labor Markets with Two-Sided Search. Amer. Econ. Rev., 90, 46-72

[16] Miettinen, T. (2009): The partially cursed and the analogy-based expectation equilibrium. Econ. Letters, 105, 162-164.

[17] Mohlin, E. (2009): Optimal Categorization. SSE/EFI Working Paper, 721, Stockholm School of Economics. 